J. Amer. Soc. Hort. ScI. 118(1):135-140. 1993.

\title{
Kernel Changes in a Shrunken2 Maize Population Associated with Selection for Increased Field Emergence
}

John A. Juvik and Maurice C. Jangulo

Department of Horticulture, University of Illinois, Urbana, IL 61801

John M. Headrick and Jerald K. Pataky

Department of Plant Pathology, University of Illinois, Urbana, IL 61801

William F. Tracy

Department of Agronomy, University of Wisconsin, Madison, WI 53706

Additional index work Zea mays, sweet corn plant breeding, principal component analysis, disease resistance

\begin{abstract}
Ten cycles of simple mass selection for increased field emergence and kernel weight in a population of shrunken2 (sh2) maize affected various kernel and seedling traits. Ten of 29 variables measured were intercorrelated and were included in the first principal factor of a principal component, factor analysis. The eight variables in factor 1 that increased with cycles of selection were: emergence and kernel weight (the two selection criteria) plant height 41 days after planting (a measure of seedling vigor), uniformity of stand, total starch content per kernel total carbohydrate content per kernel, concentration of starch, and starch content per kernel. The two variables in factor 1 that decreased were: conductivity of electrolytes that leached from imbibing seeds and symptomatic infection of kernels by fungi. Factor 1 was a "seed and seedling quality" factor. The other 19 variables formed five principal factors that primarily were "sugar," "pericarp," and "asymptomatic fungal infection" factors. These five factors and the variables from which they were formed, were not affected by selection. These results suggest that seed and seedling quality factors can be improved by selection in a $\operatorname{sh} 2$ population without affecting sweetness or tenderness. These results also suggest that although selection for increased emergence and kernel weight lowered the incidence of symptomatic infection by fungi, the population was not improved specifically for resistance to Fusarium moniliforme Sheldon.
\end{abstract}

The fresh market sweet corn industry in the United States has shown a shift in recent years from traditional hybrids with sugaryl (su) endosperm to hybrids homozygous for the $s h 2$ endosperm mutation. Shrunken2 kernels have two to three times more sucrose 20 days after pollination than sul kernels (Creech, 1965), retain higher sugar levels for longer periods after harvest (Garwood et al., 1976), and are preferred in consumer taste tests (Evensen and Boyer, 1986). Since the quality of fresh market sweet corn is closely related to sugar content (Boyer and Shannon, 1983), sh2 cultivars provide additional time to transport, process, and market sweet corn of superior quality. Nevertheless, grower acceptance of $s h 2$ hybrids has been hindered, in part, by inferior seed quality, reduced emergence, poor vigor of seedlings, and poor uniformity of stands, especially when soils are < 15C (Andrew, 1982; Kulik and Schoen, 1982; Styer et al., 1980; Wolf and White, 1975). Also, the effect of weather fluctuations on the emergence of seedlings in early plantings of sh2 corn makes it difficult for growers to determine seeding rates that maximize uniformity of stand and yield.

Several factors appear to be associated with the emergence and vigor problems of $s h 2$ genotypes. Reduced content of starch and phytoglycogen in $\operatorname{sh} 2$ kernels may not provide sufficient carbohydrate reserves for optimal rates of emergence and growth of seedlings (Wann, 1980). Elevated levels of sugars increase osmotic potential and lead to membrane and pericarp damage

Received for publication 22 July 1991. Accepted for publication 13 July 1992. This research was supported by the United States-Israel Binational Agricultural Research and Development (BARD) grant no. US-1709-89, the Illinois Agricultural Experiment Station, Urbana, as part of project 68-0323, and the College of Agriculture and Life Sciences, Univ. of Wisconsin, Madison. The cost of publishing this paper was defrayed in part by the payment of page charges. Under postal regulations, this paper therefore must be hereby marked advertisement solely to indicate this fact. from the rapid influx of water during imbibition (Simon, 1978). The dramatically reduced starch content of sh 2 kernels results in a severely collapsed endosperm, cracks in the pericarp, and air pockets between the pericarp and aleurone layers (Styer and Cantliffe, 1983). The high content of kernel sugars and leakiness of pericarps have been associated with increased conductivity of leachates from sh2 seed (Wann, 1986). Seed leachates consist primarily of sucrose, glucose, and fructose (Caplan, 1984). Thus, solute leakage during germination reduces metabolic energy available for embryo growth and may provide substrates for the growth of pathogenic microorganisms. A higher incidence of seed-borne $F$. moniliforme [teleomorph: Gibberella fijikuroi (Sawada) Wollenw.] also has been reported for sh2 genotypes (Styer and Cantliffe, 1984).

Ten cycles of mass selection in a population of sh2 maize significantly increased field emergence and kernel weight (Bell et al., 1983). Seedling emergence for the 11 composite populations from these cycles was negatively correlated with the conductivity of seed leachates (Tracy and Juvik, 1988), but was not associated with pericarp thickness (Tracy and Juvik, 1989). The 11 composite populations were not evaluated for carbohydrate content of kernels or for resistance to infection of kernels by fungi. Changes in carbohydrate content of kernels could affect quality substantially. Infection of kernels by $F$. moniliforme and other fungi could reduce emergence significantly (Headrick and Pataky, 1989; Headrick et al., 1990). Thus, selection for improved emergence may have affected other traits associated with quality or resulted in inadvertent selection for resistance. to kernel infection by $F$. moniliforme.

This paper reports on changes in the characteristics of kernels

Abbreviations: PDA, potato dextrose agar; sh2, shrunken2; su, suguryl. 
of a population of sh 2 maize selected for increased field emergence and kernel weight.

\section{Materials and Methods}

A population of maize homozygous for the sh2 allele, synthesized by M.S. Zuber, Univ. of Missouri, Columbia, provided the plant material used in this study. The population had undergone 10 cycles of simple, mass selection for improved field emergence and kernel weight. Bell et al. (1983) reported gains in field emergence averaging $3.3 \%$ per cycle.

Kernels of cycles 0 through 10 provided by Zuber were grown on Drummer silty clay loam soil (Typic Haplaquoll) in Urbana, Ill., in 1985. About 50 plants from each of the 11 cycles were sib-pollinated. Ears were harvested $\approx 60$ days after pollination, dried to $\approx 10 \%$ moisture in a forced air oven, and stored at $10 \mathrm{C}$ and $20 \%$ relative humidity $(\mathrm{RH})$. Kernels from 20 to 30 ears were used to make a balanced composite for each cycle.

Four replicates of the 1985 sib-pollinated cycles were hand planted in a randomized complete-block design in Drummer soil on the Univ. of Illinois Vegetable Crops Research Farm in Ur- bana on 18 Apr. 1986 and 15 Apr. 1987. Two hundred and 100 kernels of each cycle were planted in each of the four replicates in 1986 and 1987, respectively. Emergence, plant height (a measure of seedling vigor), and stand uniformity were recorded on 29 May 1986, 41 days after planting, and on 27 May 1987, 42 days after planting.

Including the field evaluations, 29 variables were measured for each of the 11 composite populations from each cycle of selection (Table 1). Kernels from each of the cycles generated in 1985 were proportioned into four replicates. Subsamples from each replicate were used for field and laboratory assays. Data for pericarp thickness and the conductivity of electrolyte leachate from imbibing seeds were generated as described previously (Tracy and Juvik, 1988,1989). Subsamples of 100 kernels were freeze-dried, ground into powder and assayed for sugar (fructose, glucose, sucrose, maltose, and sorbitol), phytoglycogen, and starch levels on a concentration (milligrams per gram dried kernel powder) and per kernel basis (milligrams per kernel) using the procedures described by Juvik and LaBonte (1988) and Headrick et al. (1990). Incidence of symptomatic and

Table 1. Means, SD, and ranges of 29 variables measured from a composite population of sh2 maize from each of 10 cycles of mass selection for increased emergence and kernel weight.

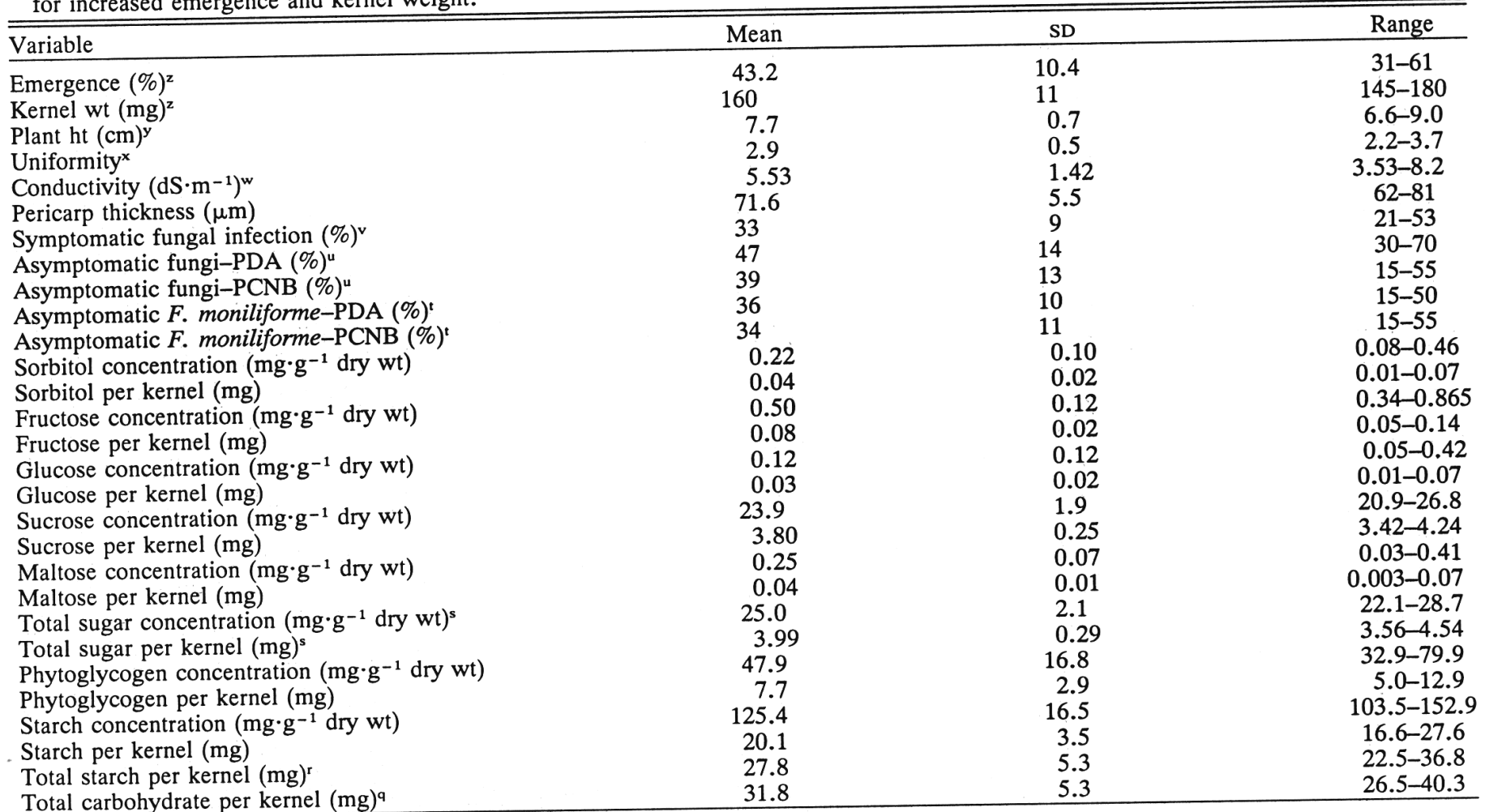

${ }^{2}$ From four replicates of 200 seeds (1986) and 4 replicates of 100 seeds (1987).

y Calculated from individual measurements of 25 plants per plot.

Where $0=0 \%-10 \%$ of the population in a plot had plant heights within $2.5 \mathrm{~cm}$ of the plot mean; $1=11 \%-20 \% ; 2=21 \%-50 \% ; 3=$ $51 \%-80 \% ; 4=81 \%-90 \%$; and $5=91 \%-100 \%$.

wConductivity of electrolyte leachate from imbibing seeds.

vercent kernels displaying visible signs of fungal infection.

uPercent of healthy appearing kernels from which fungi were isolated when seed were surface sterilized and plated on PDA or PCNB agar.

'Percent of healthy appearing kernels from which $F$. moniliforme was isolated when seed were surface sterilized and plated on PDA and PCNB

sSum of sorbitol, fructose, glucose, sucrose, and maltose in milligrams per gram dry weight and milligrams per kernel.

rSum of water soluble (photoglycogen) and water insoluble starch in milligrams per kernel.

${ }^{\mathrm{q}} \mathrm{Sum}$ of total sugar and total starch in milligrams. 

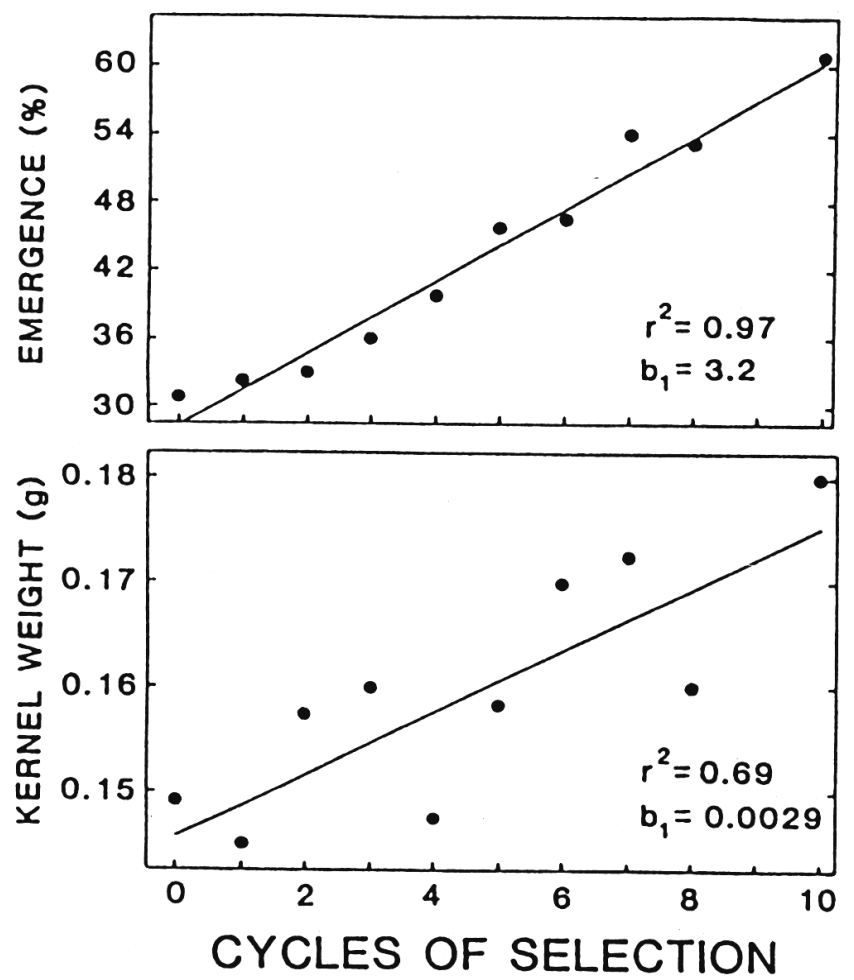

Fig. 1. Regressions of field emergence and kernel weight on cycles of selection for a population of $s h 2$ maize that had undergone 10 cycles of mass selection for those traits.

asymptomatic infection of kernels by $F$. moniliforme and other fungi was measured according to the methods described by Headrick and Pataky (1989).

Pearson's product-moment correlation coefficients were calculated among trials for the three variables measured in the field: emergence 41 days after planting, plant height 41 days after planting, and uniformity of stands. Means of the three variables were weighted by the number of replications per trial and were calculated for each composite. Grand means and SD were calculated for these three and the other 26 variables measured from the 11 composites. Associations among the 29 variables were determined by factor analysis using Varimax rotation to improve the interpretability of the solution (Green, 1978; SAS Institute, 1982). Variables were considered strongly or weakly loaded in a factor if the absolute value of the factor loading was $>0.75$ or 0.50 , respectively. The number of factors retained was determined by Cattell's Scree Test (Green, 1978) and by the ability of a factor to explain at least $5 \%$ of the total variation among the 29 variables. Pearson's product-moment correlation coefficients were calculated among the variables loaded into each factor. Correlation coefficients also were calculated among cycles of selection, the principal factors derived from the 29 variables, and the individual variables in factors that were correlated with cycles of selection. The effects of selection on the two selection criteria (field emergence and kernel weight), on the principal factors derived from the 29 variables, and on individual variables that loaded into factors correlated with cycles of selection were analyzed by least-square linear regression.

\section{Results}

Percent field emergence, plant height 41 days after planting, and uniformity of stand (as measured by coefficients of variation for emergence) were highly correlated among the 1986 and 1987 field experiments. Correlation coefficients among trials ranged from 0.82 to $0.97,0.92$ to 0.96 , and 0.59 to 0.91 for emergence, plant height, and stand uniformity, respectively. Thus, weighted means of these variables were indicative of response to selection in all trials.

Emergence ranged from $31 \%$ to $61 \%$ among the 11 composite populations from 10 cycles of selection and averaged $-43 \%$ (Table 1, Fig. 1). Likewise, kernel weight ranged from 145 to $180 \mathrm{mg}$ and averaged $\approx 160 \mathrm{mg}$ (Table 1, Fig. 1). Selection resulted in a significant linear improvement in emergence of $\approx 3.2 \%$ per cycle and increased kernel weight $\approx 3 \mathrm{mg}$ per cycle (Fig. 1).

Six principal factors accounted for $92.3 \%$ of the variation among the 29 variables (Table 2). Factor 1 was a seed and seedling quality factor and accounted for $28.8 \%$ of the variation and was comprised of nine variables: emergence, kernel weight, height of plants, uniformity, conductivity, symptomatic infection of kernels by fungi, starch content per kernel, total starch, content per kernel, and total carbohydrate content per kernel. Concentration of starch per kernel was weakly loaded in factor 1. Factor 2, a "sugar" factor, accounted for $23.7 \%$ of the variation and included sorbitol, fructose, sucrose, total sugars, and asymptomatic infection of kernels by $F$. moniliforme as determined on potato dextrose agar (PDA). Factor 3, a "glucose" factor, accounted for $11.7 \%$ of the variation. Pericarp thickness was the only variable to load strongly in factor 4 , which accounted for $10.6 \%$ of total variation. Phytoglycogen and asymptomatic infection by various fungi as determined on PDA were weakly loaded in factor 4 . Maltose loaded strongly and phytoglycogen loaded weakly in factor 5, which accounted for $9.8 \%$ of the variation. Factor 6, an "asymptomatic fungal infection" factor, accounted for $7.7 \%$ of the variation.

All variables loading strongly into a factor were highly correlated with other variables in that factor. For example, absolute values of correlation coefficients ranged from 0.57 to 0.99 among the nine variables that were strongly loaded in factor 1 (Table 3).

Factor 1 was the only principal factor associated with, cycles of selection. The linear term was significant for the regression of factor 1 on cycles of selection, and the coefficient of determination was 0.81 (Fig. 2). The slope coefficient of 0.29 represents the average gain per cycle, although residuals from regression were negative for cycles 2 through 6 and positive for cycles 7 and 8 , indicating that gains may have been greater during later cycles. Nevertheless, quadratic terms were not significant when included in the regression model. Correlation coefficients for cycles of selection and the five other principal factors were below 0.3 ; thus, selection did not substantially affect factors 2 through 6 .

In addition to the two selection criteria, emergence and kernel weight, the other eight variables in factor 1 also were highly correlated with cycles of selection (Table 3, Fig. 3). Thus, selection in this population for increased field emergence and kernel weight also affected other kernel and seedling characteristics. Plant height 41 days after planting and stand uniformity were increased significantly, while conductivity of electrolyte leachates and symptomatic infection of kernels by fungi were decreased significantly by selection for emergence and kernel weight. With respect to values in cycle 0 , change per cycle of selection was $\approx 3.4 \%, 7.1 \%,-5.4 \%$, and $-5.4 \%$ for height, uniformity, conductivity, and infection, respectively. Actual change per cycle of selection are 
Table 2. Varimax-rotated factor loadings and cumulative variance accounted for by the principal factors of 29 variables measured for a population of $\operatorname{sh} 2$ that had undergone 11 cycles of mass selection for improved field emergence and increased kernel weight.

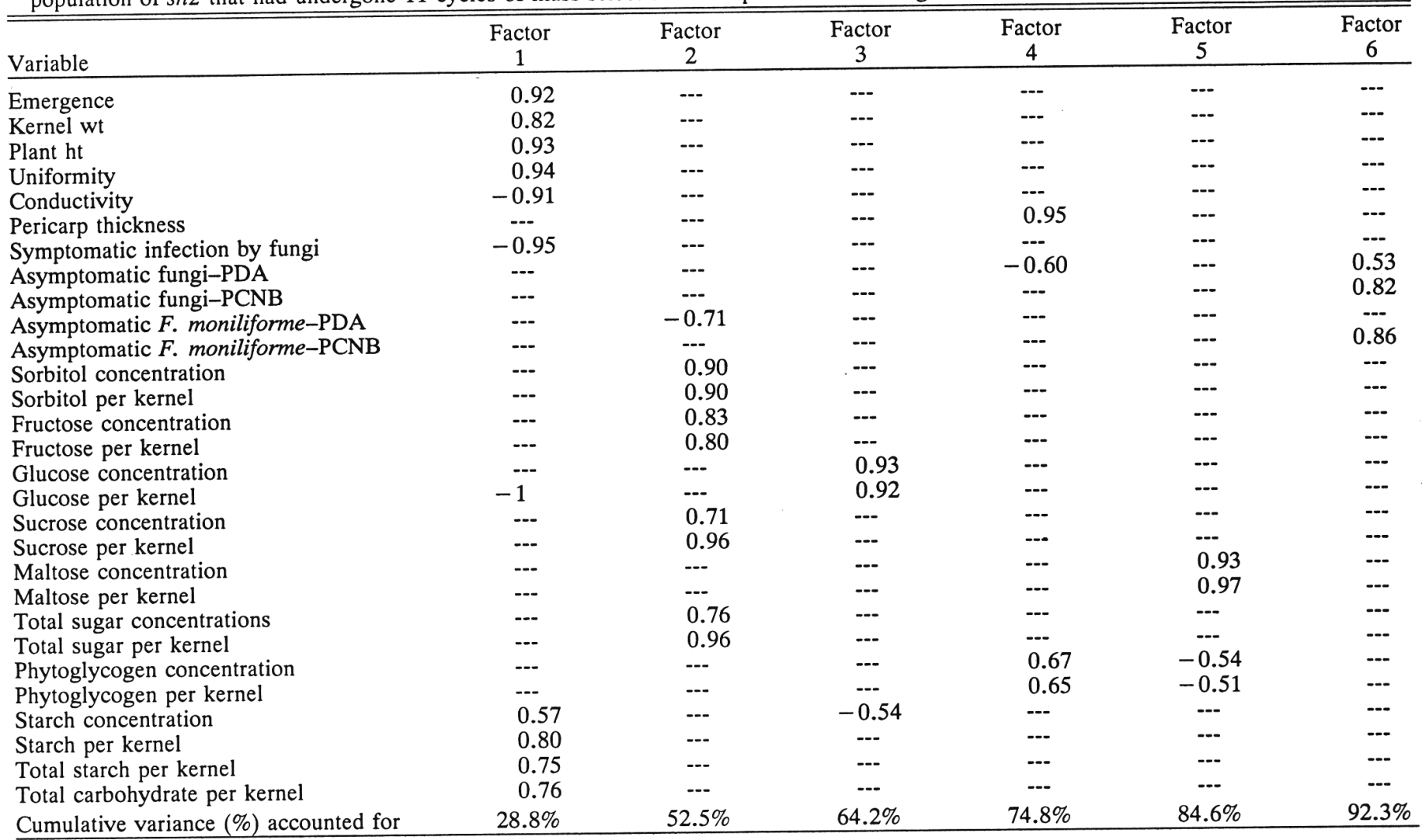

Table 3. Correlation matrix for cycles of selection and the 10 variables that loaded in the first principal factor.

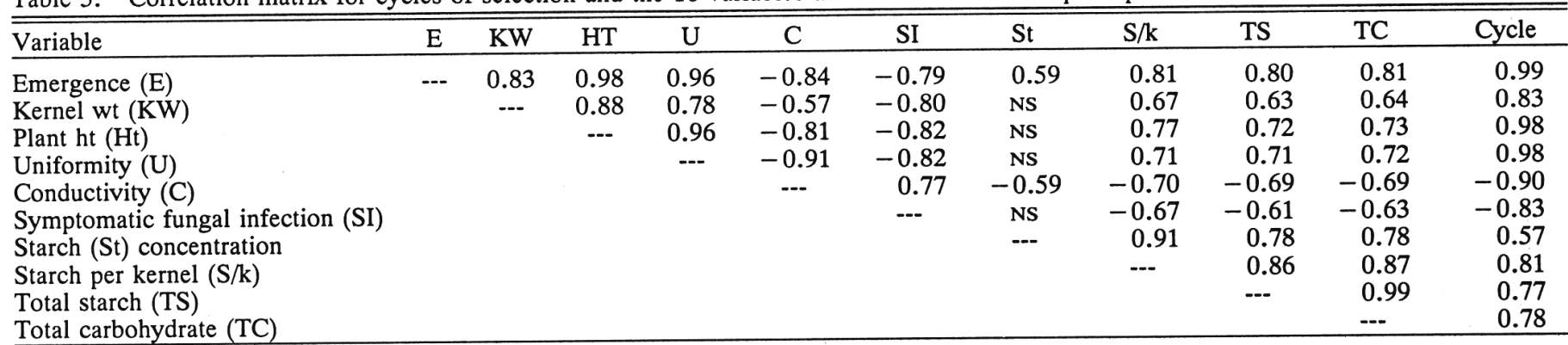

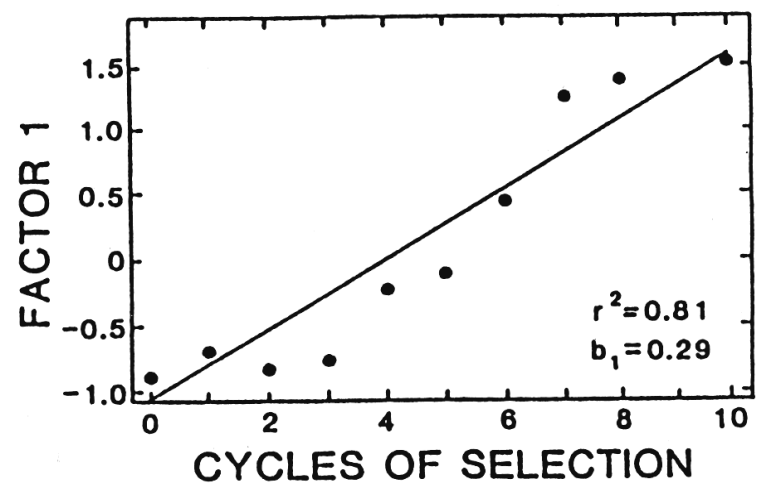

Fig. 2. Regressions of principal factor 1 on cycles of selection for population of $\operatorname{sh} 2$ maize that had undergone 10 cycles of mass st lection for increased field emergence and kernel weight. represented by the slope coefficients from the regression models (Fig. 3). The carbohydrate variables that were included in factor 1 were more variable in response to selection $\left(\mathrm{r}^{2}\right.$-values ranging from 0.33 to 0.65 ), although a significant gain per cycle was observed for all four carbohydrate variables. Carbohydrage variables tended to have negative residuals from regression for cycles 3 through 7 and positive residuals for cycles 1,7 , and 8 . In fact, starch content (milligrams) per kernel was best described by a curvilinear model where gain per cycle was substantially higher from cycles five through 10.

\section{Discussion}

Selection for increased field emergence and kernel weight in this population of sh2 maize was successful, as reported previously (Bell et al., 1983; Tracy and Juvik, 1988). In addition 

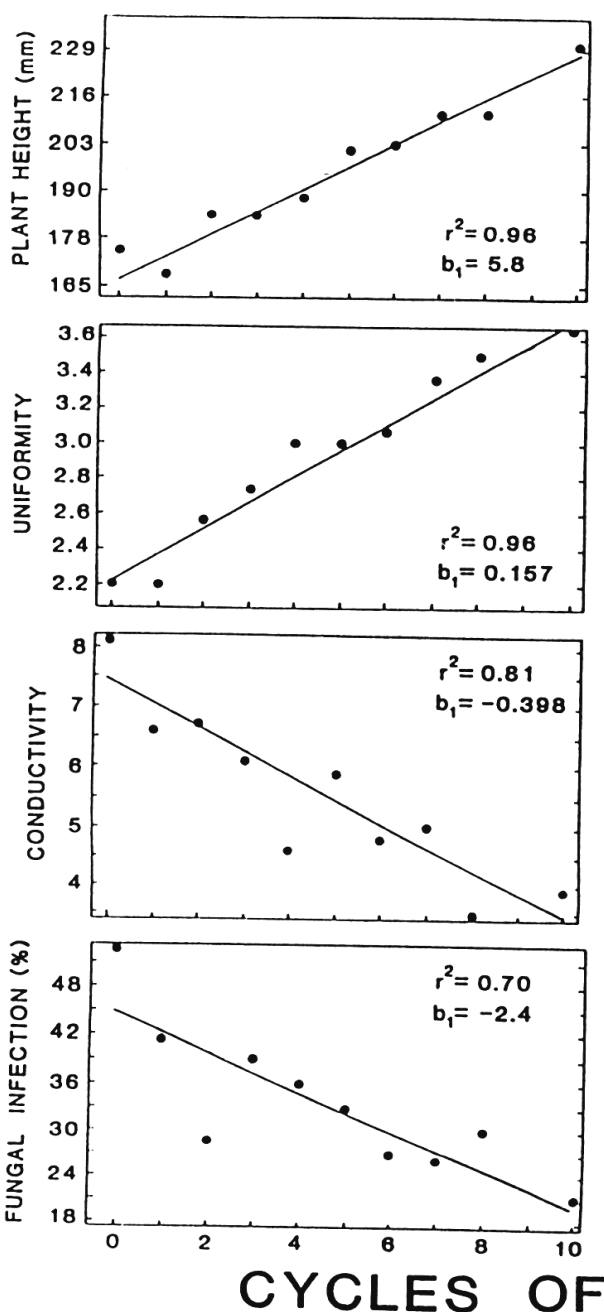

Fig. 3. Regressions of eight variables in principal factor 1 on cycles of selection for a population of sh 2 maize that had undergone 10 cycles of mass selection for increased field emergence and kernel weight.

to these two traits, several other seedling and kernel characteristics also were affected by selection. Plant height 41 days after planting (a measure of seedling vigor), stand uniformity, total starch content per kernel, total carbohydrate content per kernel, and starch content and concentration per kernel were increased by selection for increased emergence and kernel weight. Conductivity of electrolytes leached from imbibing seeds and symptomatic infection of kernels by fungi were decreased by selection for emergence and kernel weight.

The variables responding to selection were highly intercorrelated and formed the first principal factor of the factor analysis. These variables are some of the causes and effects of increased emergence and kernel weight. An increase in starch and carbohydrate content of kernels and a decrease in electrolyte leakage and fungal infection are probably associated with increases in kernel weight and emergence. Likewise, increased kernel weight and emergence are likely to increase uniformity of stands and vigor of seedlings. Thus, we designated the first principal factor a seed and seedling quality factor.

Factor 1 was the only factor correlated with cycles of selection. The other five factors, which were not correlated with selection, were primarily sugar, pericarp, and asymptomatic in-
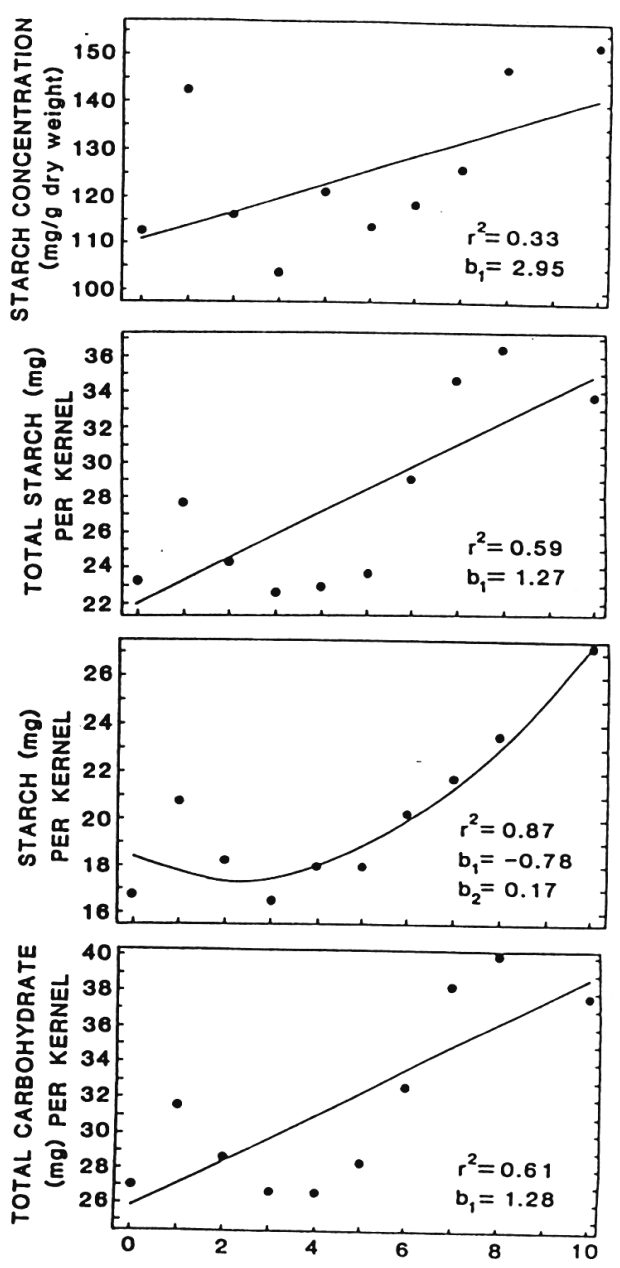

SELECTION 
seedlings that emerge normally. Although kernels were classified symptomatic based on signs and symptoms of $F$. moniliforme, such a subjective determination likely included other fungi as well; however, asymptomatic infection was determined from plating healthy-appearing kernels on agar and, thus, more accurately indicated the incidence of $F$. moniliform only. Many other fungi have been associated with poor emergence of seedlings (Koehler, 1943). Consequently, the population probably was improved for incidence of fungal infection in general, but is was not improved specifically for resistance to $F$. moniliforme, which can be under maternal influence (Headrick and Pataky, 1991).

Asymptomatic infection loaded into factor 2, a sugar factor, but other measures of fungal infection were not associated with kernel sugars. These results provide circumstantial evidence that supports a previous conclusion that incidence of fungal infection of kernels is not associated with the high sucrose content of the sh2 endosperm mutation (Headrick et al., 1990).

Many of the carbohydrate variables in factor 1 did not increase substantially until the later cycles of selection. Possibly, early gains in emergence and kernel weight were due to variables that were not measured, whereas the carbohydrate variables contributed to the gains in emergence and kernel weight made in the later cycles. It would be naive to conjecture that the variables we analyzed in this study were the only ones affecting emergence and kernel weight of this population of $\operatorname{sh} 2$ maize.

\section{Literature Cited}

Andrew, R.H. 1982. Factors influencing early seedling vigor of shrunken-2 maize. Crop Sci. 22:263-266.

Bell, R.D., L.L. Darrah, and M.S. Zuber. 1983. Progress from mass selection for field emergence and seed weight in sh2 population of maize. Crop Sci. 23:461-464.

Boyer, C.D. and J.C. Shannon. 1983. The use of endosperm genes for sweet corn quality improvement. Plant Breeding Rev. 1:139161.

Caplan, L.A. 1984. The effect of seed moisture on metabolite leakage and seedling emergence of high sugar sweet corn. MS Thesis, Univ. of Illinois, Champaign-Urbana.

Creech, R.G. 1965. Genetic control of carbohydrate synthesis in maize endosperm. Genetics 52:1175-1186.

Evensen, K.B. and C.D. Boyer. 1986. Carbohydrate composition and sensory quality of fresh and stored sweet corn. J. Amer. Hort. Sci. 111:734-738.

Garwood, D.L., F.J. McArdle, S.F. Vanderslice, and J.C. Shannon. 1976. Postharvest carbhoydrate transformations and processed quality of high sugar maize genotypes. J. Amer. Soc. Hort. Sci. 101:400404.
Green, P.E. 1978. Analyzing multivariate data. Dryden Press, Hinsdale, Ill.

Headrick, J.M. and J.K. Pataky. 1989. Resistance to kernel infection by Fusarium moniliforme in inbred lines of sweet corn and the effect of infection on emergence. Plant Dis. 73:887-892.

Headrick, J.M and J.K. Pataky. 1991. Maternal influence on the resistance of sweet corn lines to kernel infection by Fusarium moniliforme. Phytopathology 81:268-274.

Headrick, J.M., J.K. Pataky, and J.A. Juvik. 1990. Relationships among carbohydrate content of kernels, condition of silks after pollination, and the response of sweet corn inbred lines to infection of kernels by Fusarium moniliforme. Phytopathology 80:487-494.

Huelson, W.A. 1954. Sweet corn. Interscience Publ., New York. 409 $\mathrm{p}$.

Juvik, J.A. and D.R. LaBonte. 1988. Single-kernel analysis for the presence of the sugary enhancer (se) gene in sweet corn. HortScience 23:384-386.

Koehler, B. 1942. Natural mode of entrance of fungi into corn ears and some symptoms that indicate infection. J. Agr. Res. 64:421442.

Kulik, M.M. and J.F. Schoen. 1982. Germination, vigor and field emergence of sweet corn seeds infected by Fusarium moniliforme. Seed Sci. Technol. 10:595-604.

SAS Institute 1982. User's Guide: Statistics. SAS Institute, Cary, N.C. Simon, E.W. 1978. Plant membranes under dry conditions. Pest Sci. 9:168-172.

Simon, E.W. 1984. Phospholipids and plant membrane permeability. New Phytol. 377-420.

Styer, R.C. and D.J. Cantliffe. 1983. Relationship between environment during seed development and seed vigor of two endosperm mutants of corn. J. Amer. Soc. Hort. Sci. 108:717-720.

Styer, R.C. and D.J. Cantliffe. 1984. Infection of two endosperm mutants of sweet corn by Fusarium moniliforme and its effect on seedling vigor. Phytopathology 74:189-194.

Styer, R.C., D.J. Cantliffe, and L.C. Hannah. 1980. Differential seed and seedling vigor in shrunken-2 compared to three other genotypes of corn at various stages of development. J. Amer. Soc. Hort. Sci. 105:329-332.

Tracy, W.F. and J.A. Juvik. 1988. Pericarp thickness of a shunken2 population of maize selected for improved field emergence. Crop Sci. 29:72-74

Tracy, W.F. and J.A. Juvik. 1989. Electrolyte leakage and seed quality in a shrunken-2 maize selected for improved field emergence. HortScience 23:391-392.

Warm, E.V. 1980. Seed vigor and respiration of maize kernels with different endosperm genotyps. J. Amer. Soc. Hort. Sci. 105:3134.

Wann, E.V. 1986. Leaching of metabolites during imbibition of sweet corn seed of different endosperm genotypes. Crop Sci. 26:731-734.

Wolf, E.A. and J.M. White. 1975. Improved germination and plant stand of "Florida Sweet" corn by seed treatment. Proc. Fla. State Hort. Soc. 88:170-173. 